\title{
Adaptive Inverse System Control of Electromagnetic Linear Actuator
}

\author{
Zhu Jianhui and Chang Siqin \\ School of Mechanical Engineering, Nanjing University of Science and \\ Technology, Nanjing 210094 China \\ ffxyz505@gmail.com
}

\begin{abstract}
Against EMLA (Electromagnetic Linear Actuator) in the long-running, the changed parameters of actuator coil resistance and inductance caused by temperature rise resulted in degradation of control performance, and parameters recondition in the inverse system control program. By using parameter identification method based on recursive least squares method (RLSM), the adaptive inverse system control is implemented in electromagnetic linear actuator. Under Matlab/Simulink it establishes the simulation model to simulate online identification process and the adaptive inverse control system strategy which achieve the control effect when the parameters change, and the test data are identified off-line verification. The results show that it is able to accurately identify the actuator coil resistance and inductance parameters, and at the same time, it can identify system's dynamic mass, viscous friction coefficient and disturbance force of load. Finally, the test platform was built to verify, the identification results was used to control parameters online real-time. And the parameters identification was used in the practical application of inverse system control, implemented system's adaptive control, improved system's robustness in long run.
\end{abstract}

Keywords: Electromagnetic Linear Actuator, Parameter Identification, Adaptive Control, Inverse System, Least Square Method

\section{Introduction}

EMLA has been widely used because of its high performance, high response, and the characteristics of high precision linear servo position, such as, direct drive servo valve, electromagnetic valve mechanism, and AMT mechanism etc. At the same time, it also put forward higher requirements for its control [1]. When EMLA runs in the long process, due to change of the load, viscous friction coefficient change and disturbance, EMLA's controller performance is declined, and even caused instability in the optimized system. Therefore, how to eliminate its load change and the impact of disturbance while EMLA in the run process, how to improve the robustness of electromagnetic linear actuator control system has become an urgent problem to be solved.

With the method of parameter identification, and through the online real-time recognize to identify the system current parameters, and then pass the real value to system, and make the system adaptation control according to the current parameters, in order to get a better control performance [2,3]. At present, parameter estimation is mostly used for permanent magnet synchronous motor (PMSM) [4]. Such as bibliography [5, 6] which based on least square method (LMS) and Kalman algorithm is to do online parameter identification for electronic resistance motor, inductance and magnetic linkage. And bibliography [7, 8] identified induction motor rotor time constant. And bibliography [9] researched the speed control of induction motor. Moreover, there is bibliography [10] presented a method for estimating overhead line parameters. The above identification methods, most of them are based on the magnetic chain model, or motor rate to do vector 
control. However, there is seldom research for identification of EMLA's nonlinear system model parameters and adaptive inverse control.

This article carries on decoupling control with the principle of inverse system, and using feedback linearization to decouple multivariable, nonlinear, strong coupling system. The nonlinear system is transformed into a linear system, and combined with the method of parameter identification to realize the adaptive adjustment system.

\section{The Establishment and Simulation of Identification Model}

\subsection{The Parameter Identification Theory of Least Square Method}

Figure 1 is the most basic form of expression for LMS. The principle is parameters for a given system; calculate model's state variables and observation vector, and make comparisons between the observation value and the actual output values of system, calculate sum of squared error. And then through a certain way to constantly modify the parameter values, when the sum of squared error is at the minimum, the parameter matrix of system is identified, and makes the obtained parameter as the closest value of the actual system parameters.

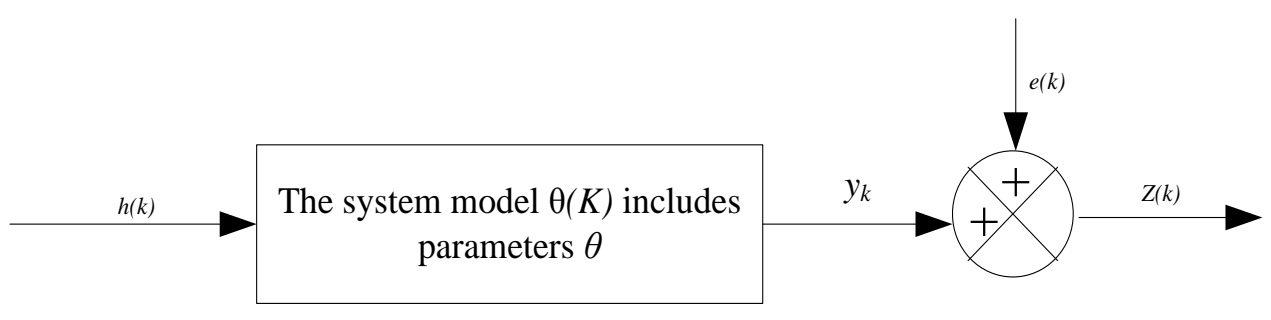

Figure 1. Expressed Forms of Least Squares Method

In the Figure $1, h(k)$ is the input, $y(k)$ is the output, $e(k)$ is the test noise, $z(k)$ is the output of measurement, $\theta(k)$ is the unknown model parameter.

$$
\begin{gathered}
h(k)=\left[h_{1}(k), h_{2}(k), \ldots h_{n}(k)\right]^{T} \\
\theta=\left[\theta_{1}, \theta_{2}, \ldots \theta_{n}\right]^{T}
\end{gathered}
$$

The model output is:

$$
z(k)=h^{T}(k) \theta+e(k)
$$

According to the definition of least squares method, it can deduce the method of least squares criterion function is:

$$
J(\theta)=\sum_{k=1}^{n}\left[z(k)-h^{T}(k) \theta\right]^{2}
$$

So as to minimize $J(\theta)$, the estimation value of $\theta$ as $\hat{\theta}$ is called the least square estimation of parameter values. The derivation of expression for the recursive least squares method is:

$$
\left\{\begin{array}{l}
\hat{\theta}(k)=\left[h(k)^{T} h(k)\right]^{-1} h(k)^{T} z(k) \\
P(k)^{-1}=h(k)^{T} h(k)
\end{array}\right.
$$


The following recursive formula is derived:

$$
\begin{gathered}
\hat{\theta}(k)=\hat{\theta}(k-1)+K(k)\left[y(k)-\varphi^{T} \hat{\theta}(k-1)\right] \\
K(k)=\frac{P(k-1) \varphi(k)}{1+\varphi^{T}(k) P(k-1) \varphi(k)} \\
P(k)=\left[I-K(k) \varphi^{T}\right] P(k-1) \\
J(k)=J(k-1)+\frac{\left[z(k-1)-h^{T}(k-1) \theta(k-1)\right]^{2}}{1+\varphi^{T}(k-1) P(k-1) \varphi(k-1)}
\end{gathered}
$$

Thus the calculation formula for the residual is:

$$
\varepsilon(k)=\frac{\left[z(k-1)-h^{T}(k-1) \theta(k-1)\right]^{2}}{1+\varphi^{T}(k-1) P(k-1) \varphi(k-1)}
$$

Of which $K(k)$ is the gain matrix going to zero with the increase of $y^{2}, P(k)$ is the decreasing positive definite symmetric matrix with the increase of $y$.

While using the above recursive formula for calculation, the initial value $P(0)$ and $\hat{\theta}(0)$ determine the needs. Generally, $P(0)=\alpha I, \hat{\theta}(0)=\varepsilon$, of which $I$ is the unit matrix, $\alpha$ is a sufficiently large arithmetic number $\left(10^{4}-10^{6}\right), \varepsilon$ is the zero vector. By choosing the appropriate initial value, after recursive operation, the residual error $\varepsilon(k)$ is less than the appropriate small real number; it is namely thought criterion function achieves a minimum value, identification process is over.

\subsection{The Parameter Estimation of System}

Electromagnetic linear actuator is a nonlinear, strong-coupling, and single-input-singleoutput system (SISO). To achieve the object's approximate decoupling, the voltage balance equation of electromagnetic linear actuator at steady-state is:

$$
U=I R+L \frac{d I}{d t}+E_{e m f}
$$

In the Equation 11, $U$ is the supply voltage, $I$ is the coil current, $R$ is coil impedance, $L$ is the coil inductance, $E_{e m f}$ is the back electromotive force (EMF) in the coil, $E_{e m f}=k_{m} v, K_{m}$ is the EMF constant, $v$ is rotor velocity.

To achieve the adaptive parameter identification by using the recursive algorithm of least squares parameter method identification, Equation (11) is rewritten into the expression form of LMS:

$$
u=\left[\begin{array}{lll}
I & I & k_{\mathrm{m}}
\end{array}\right]\left[\begin{array}{lll}
R & L & 1
\end{array}\right]^{T}
$$

Of which input matrix is $h(k)=\left[I(k) \dot{I}(k) k_{\mathrm{m}} v(k)\right]$, the parameter matrix is $\hat{\theta}(k)=\left[\begin{array}{lll}R & L & 1\end{array}\right]^{T}$ the output is $u$. In the input quantity, current differential is needed and discretized. 


$$
\dot{I}=[I(k)-I(k-1)] / T_{s}
$$

In the Equation 13, $T_{s}$ is the sampling period, and $T_{s}=1 \times 10^{-4} \mathrm{~S}$.

The structure and parameter of controller is able to make the appropriate adjustments according to the change of load. According to the motion equations of motor $F=m a$, under the constant thrust provided by motor, system which changes in mass of movement have the greatest effect of control performance, so the different movement mass needs to be identified. The parameters of the system are auto-adjusted and optimized according to the identification results. Meanwhile, because in the case of the motor keeping in the long run, viscous friction coefficient will also change which needs to be identified and regulated according to different states.

According to the EMLA, differential equation of motion is:

$$
m \dot{v}=F_{m}-B v-F_{l}
$$

In the Equation (14), $m$ is the motion mass, $v$ is the velocity of moving part, $F_{m}$ is the electromagnetic force, $B$ is the viscous friction coefficient, $F_{l}$ is the load resistance.

In order to facilitate the practical digital control, Equation (14) is discretized. In the inputs of a control object, and add zero-order hold $(\mathrm{ZOH})$ to maintain a constant value, thus Equation (14) has $\mathrm{Z}$ transformation and discretization which is converted into:

$$
\begin{gathered}
V(z)_{Z O H}=\left(1-z^{-1}\right) Z(V(s) / s) \\
v(k)=e^{-B T_{s / m}} v(k-1)+\frac{\left(1-e^{-B T_{s / m}}\right)}{B} F_{m}(k-1)-\frac{\left(1-e^{-B T_{s / m}}\right)}{B} F_{l}(k-1)
\end{gathered}
$$

According to $F_{m}=I K_{m}, a=-e^{-B T_{s} / m}, b=(1+a) K_{m} / B, c=b F_{l}(k-1) / K_{m}$, Equation (16) can be transformed to:

$$
v(k)=-a v(k-1)+b I(k-1)-c
$$

With reference to the form of LMS, Equation (17) is converted into:

$$
\begin{aligned}
& h(k)=\left[\begin{array}{lll}
-v(k-1) & I(k-1) & -1
\end{array}\right]^{T} \\
& \hat{\theta}=\left[\begin{array}{lll}
\hat{a} & \hat{b} & \hat{c}
\end{array}\right]
\end{aligned}
$$

In the Equation (19), $h(k)$ is the input matrix, $\hat{\theta}$ is the parameter matrix, $\hat{a}, \hat{b}, \hat{c}$ is respectively estimated the value of $a, b, c$. Identification was carried out by using the LMS of the previous section, the load resistance can be regarded as unchanged in a sampling frequency, namely: $F_{l}(k-1)=F_{l}(k)$, the following equation can be obtained.

$$
\left\{\begin{array}{l}
\hat{F_{l}}(k)=\hat{c} K_{m} / \hat{b} \\
\hat{B}=\left((1+\hat{a}) K_{m}\right) / \hat{b} \\
\hat{m}=-\hat{B} T_{s} / \ln (-\hat{a})
\end{array}\right.
$$


In the Equation (20), $\hat{F}_{l}(k), \hat{B}, \hat{m}$ is respectively the estimated value of $F_{l}, B, m$.

\subsection{Identification Results}

Under Matlab/Simulink, simulation model of system parameters identification is built. Structure diagram is shown in the following Figure 2. The whole system is divided into independent sub-module, which includes controller, controlled object, and the parameter identification module of LMS, parameter calculation module and output display. With the combination of these functional modules, the parameter on-line identification is achieved under the simulation of Simulink. The variable of input target $r$ is position signal. In the case of without control strategy, simulation and modeling of the system parameter estimation is conducted to verify the designed identification algorithm's feasibility and accuracy.

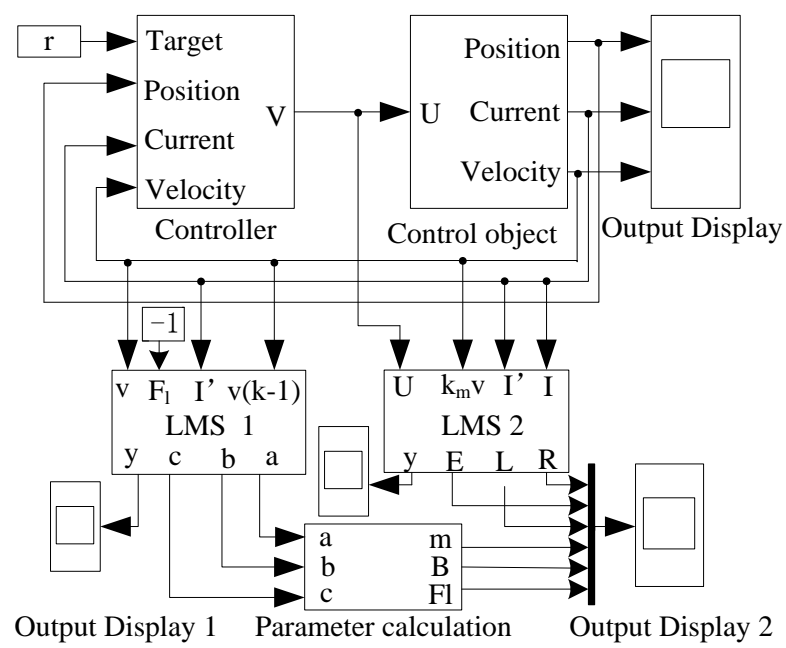

Figure 2. Simulation Model of EMLA System Parameter Identification

In simulation, the parameter of electromagnetic linear actuator is shown as Table 1.

Table 1. EMLA Parameters in the Model Simulation

\begin{tabular}{ll}
\hline Parameter Name / Unit & Parameter Value \\
\hline Supply Voltage U/v & 24 \\
Motor Resistance $\mathrm{R} / \Omega$ & 4 \\
Motor Inductance $\mathrm{L} / \mathrm{mH}$ & 1.6 \\
Damping Factor c/(Ns/m) & 2 \\
Counter-EMF Km & 10.8 \\
Rotor Mass m/Kg & 0.036 \\
\hline
\end{tabular}

The system parameter identification results are shown as in Table 2. It can be seen from Table 2: (1) When not loading the interference force, the identification error of dynamic mass is $0.0001 \mathrm{~kg}$, and the maximum error of friction coefficient identification is $0.686 \mathrm{Ns} / \mathrm{m}$, the minimum error is $0.127 \mathrm{Ns} / \mathrm{m}$, identification of disturbance force can be approximated to 0 ; (2) When loading the interference force, separately the force of $\pm 10 \mathrm{~N}$ and $\pm 20 \mathrm{~N}$, and the identification error is $0-0.02 \mathrm{~N}$ and $0-0.03 \mathrm{~N}$, and can accurately identify the disturbance force value, and provides a compensatory basis for the further control scheme, and compensates according to the obtained interference force. At the same time, it can be seen from Table 2, in the identification of disturbance force, due to the applied interference force, it makes the identification of coefficient friction also change. Because 
of the loading positive force disturbance, equivalent to increase force and thrust opposite to the system, equivalent to disguised increase friction of the system, so the friction coefficient increases. While applying a negative interference force, thus the process is opposite. So it is necessary to design a related adaptive compensation algorithm with the purpose of compensation control for the original system, to improve the accuracy of control parameters. Compensation algorithm is as follows:

$$
\begin{aligned}
\Delta i & =\Delta F_{m} / k_{m} \\
& =\left[\Delta F_{l}(k)+\Delta B \hat{v}(k)+\Delta m\left(\frac{\hat{v}(k)-\hat{v}(k-1)}{T_{s}}\right)\right] / k_{m} \\
& =\left[\left(\hat{F}_{l}(k)-F_{z}\right)+\left(\hat{B}-B_{z}\right) \hat{v}(k)+\left(\hat{M}-M_{z}\right)\left(\frac{\hat{v}(k)-\hat{v}(k-1)}{T_{s}}\right)\right] / k_{m}
\end{aligned}
$$

\begin{tabular}{|c|c|c|c|c|}
\hline $\begin{array}{l}\text { External } \\
\text { Conditio } \\
\mathrm{n}\end{array}$ & $\begin{array}{l}\text { Real Mass m } \\
(\mathrm{Kg})\end{array}$ & $\begin{array}{l}\text { Identification } \\
\text { Mass m }(\mathrm{Kg})\end{array}$ & $\begin{array}{l}\text { Identification of } \\
\text { B ( Ns/m) }\end{array}$ & $\begin{array}{l}\text { Identification } \\
\text { Disturbing Force } \\
(\mathrm{N})\end{array}$ \\
\hline \multirow[t]{3}{*}{$(1)^{*}$} & $\mathrm{~m}=0.036$ & 0.0361 & 2.345 & $-5.64 \mathrm{e}-11$ \\
\hline & $\mathrm{m}=0.054(1.5 \mathrm{~m}$ & 0.0541 & 2.164 & $-3.734 \mathrm{e}-11$ \\
\hline & $\mathrm{m}=0.72(2 \mathrm{~m})$ & 0.07211 & 2.127 & $-2.753 e-11$ \\
\hline \multirow[t]{5}{*}{$(2)^{*}$} & $\begin{array}{l}\text { Disturbance } \\
\text { Force } \\
\text { Loading Fl (N) }\end{array}$ & $\begin{array}{l}\text { Identification } \\
\text { of } \mathrm{Fl}(\mathrm{N})\end{array}$ & $\begin{array}{l}\text { Identification } \\
\text { Mass } \\
\end{array}$ & $\begin{array}{c}\text { Identification of } \\
\text { B ( Ns/m) }\end{array}$ \\
\hline & +10 & 9.98 & 0.03666 & -5.077 \\
\hline & -10 & -10 & 0.3621 & 0.02072 \\
\hline & +20 & 19.97 & 0.03685 & -6.985 \\
\hline & -20 & -20 & 0.03613 & +0.7154 \\
\hline \multirow[t]{6}{*}{$(3)^{*}$} & Real & Identification & Identification & Identification \\
\hline & Coefficient & of B ( Ns/m) & mass & Disturbing \\
\hline & Friction ( $\mathrm{Ns} / \mathrm{m}$ ) & & $\mathrm{M}(\mathrm{Kg})$ & Force $(\mathrm{N})$ \\
\hline & 2 & 2.345 & 0.361 & $-5.64 \mathrm{e}-11$ \\
\hline & 3 & 3.516 & 0.3614 & $-8.469 \mathrm{e}-11$ \\
\hline & 4 & 4.686 & 0.3619 & $-1.13 \mathrm{e}-10$ \\
\hline
\end{tabular}

\section{Table 2. Identification Results of System Parameters under Different External Conditions}

*(1) Unload disturbance force $(\mathrm{Fl}=0, \mathrm{~B}=2)$, identification of mass.

*(2) Load disturbance force $(\mathrm{m}=0.036, \mathrm{~B}=2)$, identification of disturbing force.

*(3) Unload disturbing force $(\mathrm{Fl}=0, \mathrm{~m}=0.036)$, identification of coefficient friction.

From the above analysis, it can be seen that the recursive application of LMS used in electromagnetic linear actuator is feasible, and the parameters of system and controller can be identified accurately. Moreover, the accuracy of identification is high. While the distinguished result for resistance and inductance of ELMA is shown as Figure 3 and 4: 


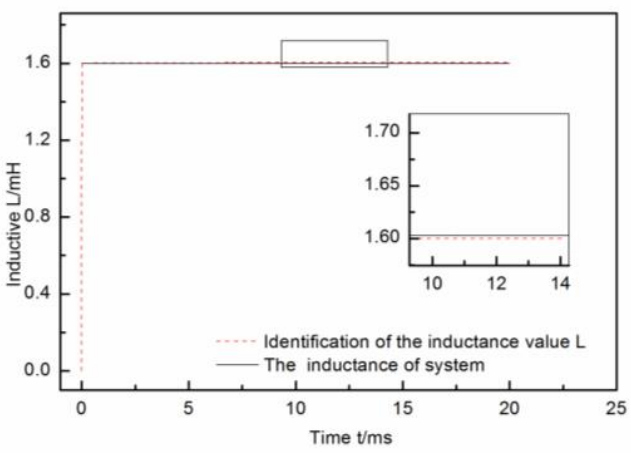

Figure 3. Identification Results of Inductive

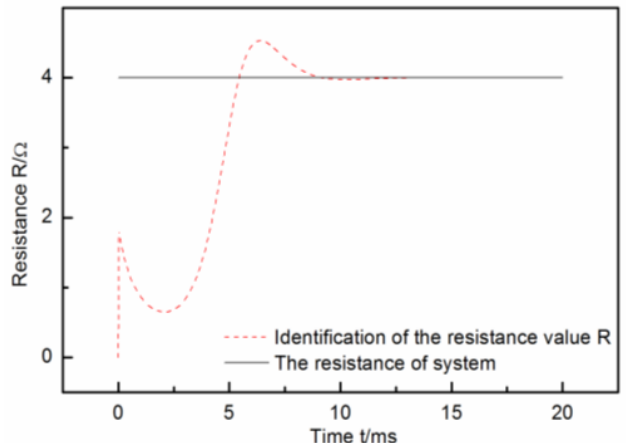

Figure 4. Identification Results of Resistance

It can be seen from Figure $3 \& 4$ that motor parameters identified by LMS quickly converge to the actual resistance and inductance value from its initial value 0 , and keep closer to the true value, finally keep in steady state.

\section{Adaptive Inverse System Control}

\subsection{The Principle of Inverse System}

The inverse system is with the method of nonlinear feedback or dynamic compensation to realize the decoupling transformation of nonlinear system into a linear system [1]. By the definition of inverse system, suppose the nonlinear system is $\Sigma^{-1}$, The initial state of the system is $x\left(t_{0}\right)=x_{0}$, description for systems $\Sigma^{-1}$ from input into output operator is $\hat{\theta}_{\alpha}$, in addition to $\varphi$ is any continuous function in a domain. $\varphi$ is mapped to $u$, take recorded $\varphi=y^{(\alpha)}$ as:

$$
y=\theta u=\theta \hat{\theta}_{\alpha} \varphi=\theta \hat{\theta}_{\alpha} y^{(\alpha)}
$$

Then calls $\Sigma^{-1}$ for $\alpha$ an integral inverse system of $\Sigma$ and $\Sigma$ is original system. With the inverse system and the original system connected in series, thus realizes decoupling process of nonlinear system. The system after reconstruction is called "pseudo linear system", which is controlled according to the linear system theory. The control system of electromagnetic linear actuator can be regarded as a single-input and single-output system. The input variable is supply voltage $u$, and finally derives the expression of $u$ as follows: 


$$
u=\frac{m L}{k_{m}} S^{\prime \prime \prime}+\frac{B L}{k_{m}} S^{\prime \prime}+k_{m} S^{\prime}+I R
$$

Equation (23) is the inverse system equation. As the controlled variable has timevarying parameters $m, L, B, R$ can be seen. Therefore, in the practical control the control volume can be modified in real time with the identified parameters, makes the inverse system is more close to the actual control system.

\subsection{The Adaptive Inverse System Controller}

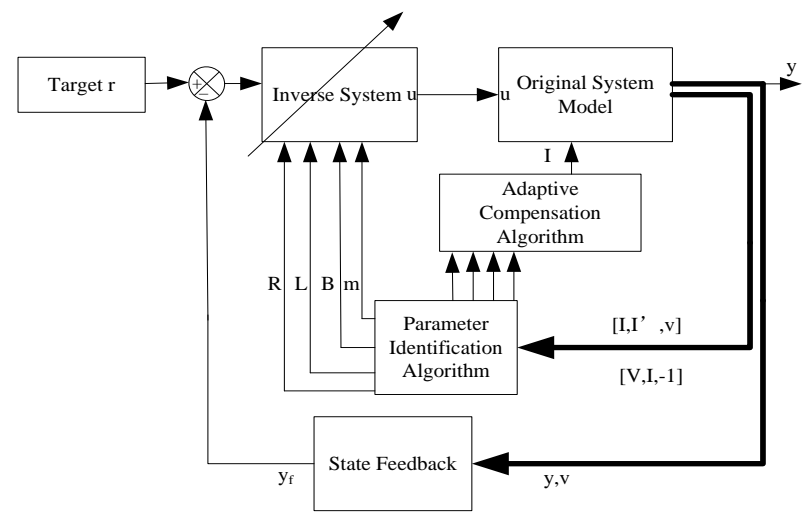

\section{Figure 5. Block Diagram of Adaptive Inverse Control Based on Parameter Identification}

Structure of the adaptive system inverse controller is as shown in Figure 5 and mainly composed of inverse system, the original system model, adaptive compensation algorithm module, parameter identification algorithm, and state feedback. The above structural parameter identification algorithm is collected into S-function, and makes an independent module under Matalb/Simulink, and forms the inverse system pseudo linear system and the original system in series, takes advantage of the theory of linear system to treat the position signal output as the state feedback, forms closed loop control. Through the parameter after parameter identification transfer to the inverse system, it revises the control parameters of the system in real time. Meanwhile, by the adaptive compensation algorithm, it loads the calculated compensating current to the model of the original system.

\subsection{Simulation Result}

For the controlled object EMLA, system parameter changes with the effect of temperature rise in the long run. Resistance is the most critical parameter effect on actuator control. While the actuator load also changes frequently in practical application, so the dynamic mass changes also affect the system control performance. By simulation calculations for the key parameters of system affect, it verifies adaptive control effect when coil resistance $R$ and moving mass $m$ changes and ignores the inductance $L$ of which influence is not very obvious and the change of viscous friction coefficient $B$. The simulation results are shown in Figure 6.

The resistance increased because of the rising temperature, and thus causes the situation of control effect becoming worse, the simulation was given. By the output effect of adaptive inverse control parameters, the simulation resistance value increased by $50 \%$. It compares the control effects of the following three kinds of circumstances: 1) No change in parameters 2) the change of parameters but the control parameters of inverse system controller not change 3 ) the controller parameters adjust control parameters 
according to the identification result when parameters change.

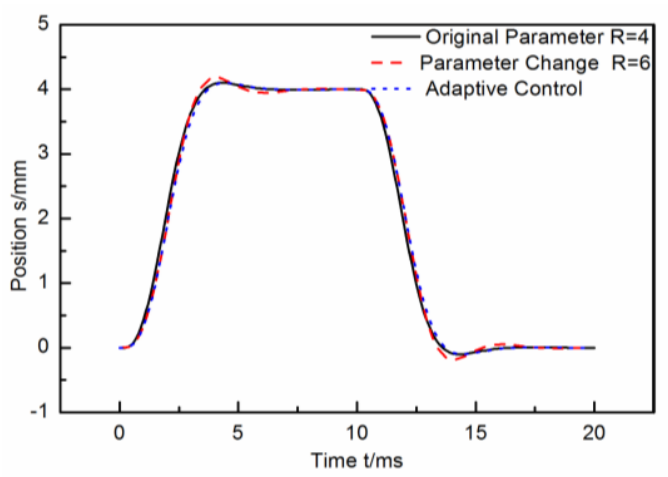

a) Position Output of Resistance Changed

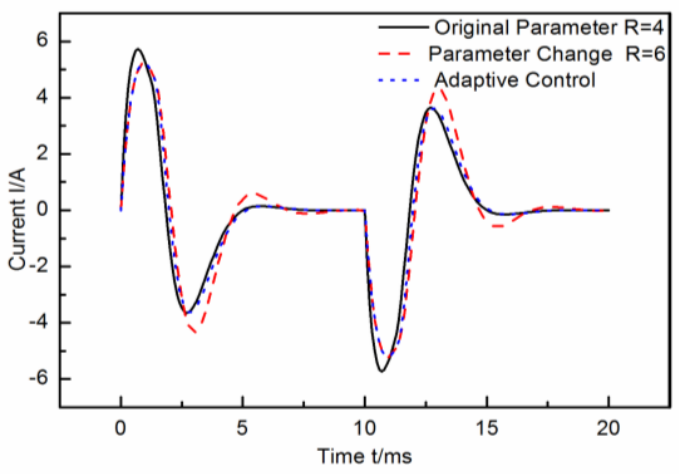

b) Current Output of Resistance Changed

Figure 6. Adaptive Control Results under Resistance Changed

Figure 6 shows that the more resistance increased, the system responses slower, and time to reach the target position becomes longer. In the case of resistance increased by $50 \%$, through the parameter identified by self-adaption, inverse system control parameters were adjusted, which can make the overshoot reduce and reach the target position quickly. Seen from the Figure 6a) and 6b), the increase of resistance makes the actuator movement velocity reduce, because the resistance directly affects the dynamic current of the stator coil, makes the current reduce, affects performance of the whole system, but by adjustment of adaptive parameters, can make the system return to steady state as soon as possible, can decrease the effect of parameter change bring to the system. 


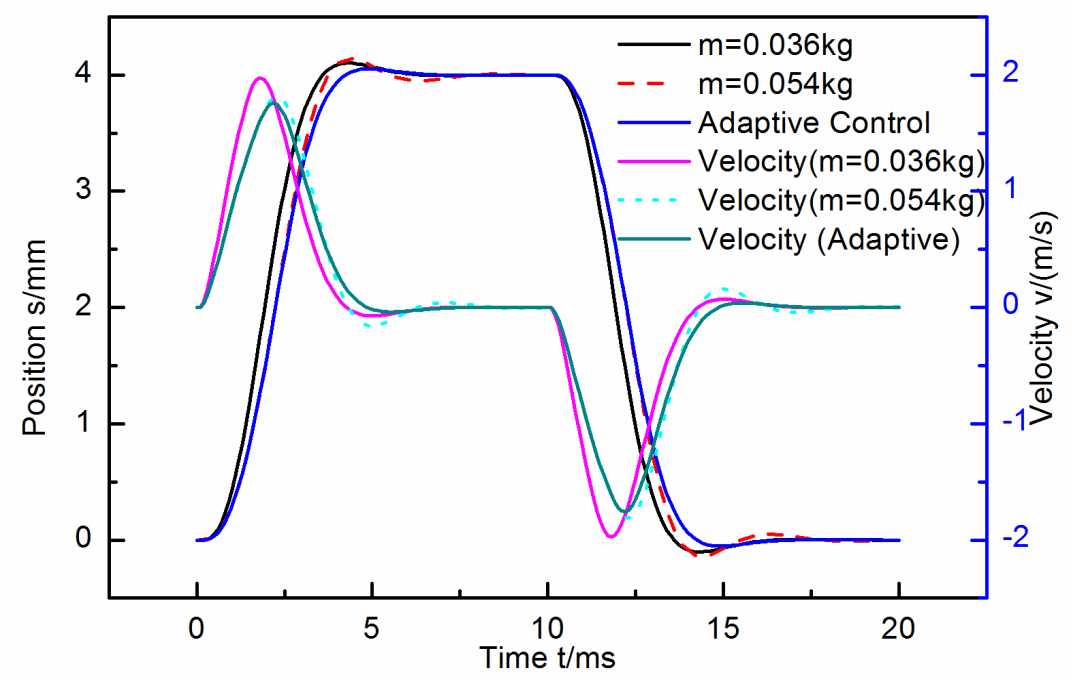

Figure 7. Adaptive Inverse System Control of Moving Mass Changed

Figure 7 is the results after dynamic mass in the system parameters increased by $50 \%$, response of position and velocity curve through the identification adaptive after control is shown in the figure. It can be seen from the Figure 7 that the increase of dynamic mass makes the system response slow, overshooting and oscillation, the system restored to the target value is slow, movement velocity is reduced. By adaptive control, the system oscillation reduces, and it can rapidly reach the target position.

\section{Experimental Verification}

In order to further verify the accuracy of the parameters identification and the effectiveness of adaptive inverse control system, it builds the adaptive inverse system control test platform of electromagnetic linear actuator (shown as Figure 8). After the simulation analysis, the test is to identify the most critical parameter for resistance, and the mass of the same actuator is relatively constant. Test device is shown as below, using resistance tester to test the initial resistance of EMLA, displacement sensor detects perform linear displacement device in real time, while the sampling frequency is $10 \mathrm{KHz}$, thus to calculate the velocity of movement through the software, and current sensor is used to test current in the run process. The sampling of displacement and electric current signal are transferred to DSP, and calculates algorithm identification, thus to obtain the practical resistance value.

During the test, EMLA runs 100 hours in the cycle of 40ms under control voltage of $24 \mathrm{~V}$. Under the circumstance of the control algorithm without loaded adaptive, it makes use of the current sensor and displacement sensor to collect current signal and position signal. The result is shown as Figure 9, after EMLA runing a long time, current peak is decreased compared with the initial value, from $7.96 \mathrm{~A}$ to $7.45 \mathrm{~A}$, while the displacement response time is increased, and the velocity of reach the target position is slowed down. It is because motor runs in a long time, the resistance increased with the rising of temperature, and which matches the change trend calculated by simulation. 


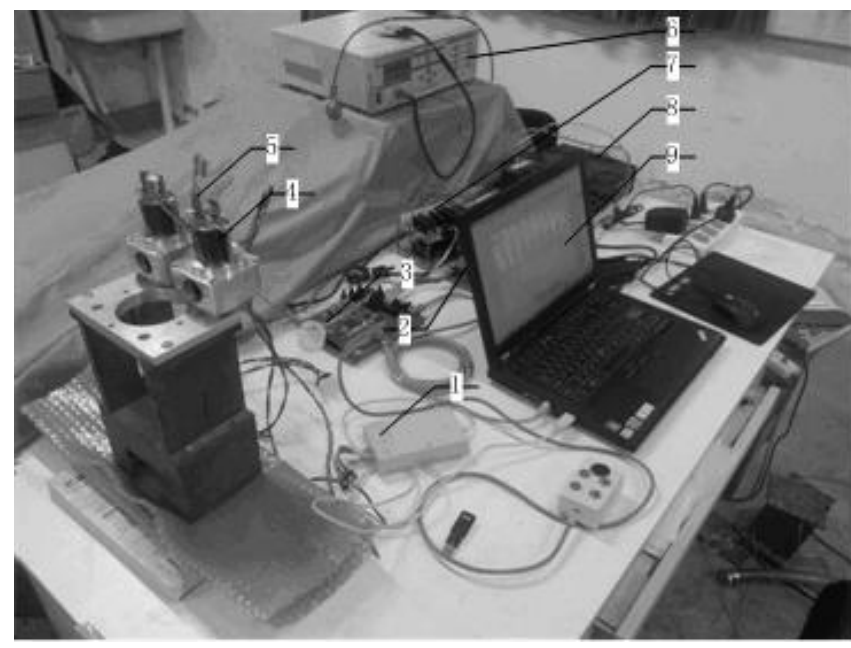

1. Simulator

2. Current Sensor

3. DSP Controller

4. EMLA

5. Displacement Sensor

6. Resistance Tester

7. Power Driver

8. Power

9. PC

Figure 8. Test Platform of Adaptive Inverse Control

To calculate the test data off-line, it needs to make comparative analysis between identified parameter and practical parameter. While the initial resistance is $0.45 \Omega$, the resistance values derived from identification algorithm is $0.57 \Omega$. The change of resistance makes the decline of the control strategy performance which based on inverse system, the position relative to the initial has a little deviation, and the response slows.

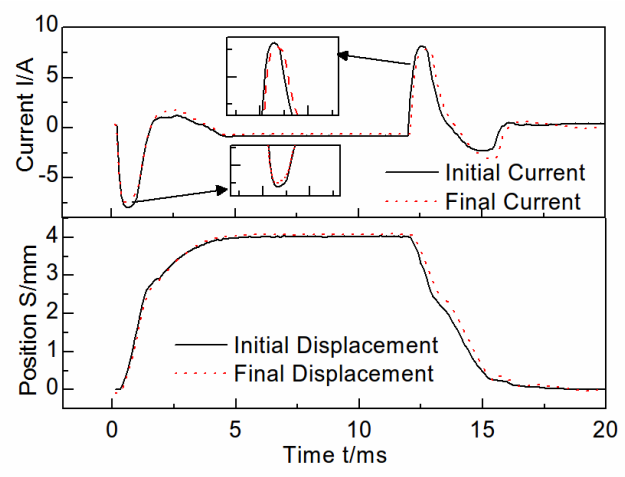

Figure 9. The Experiment Results

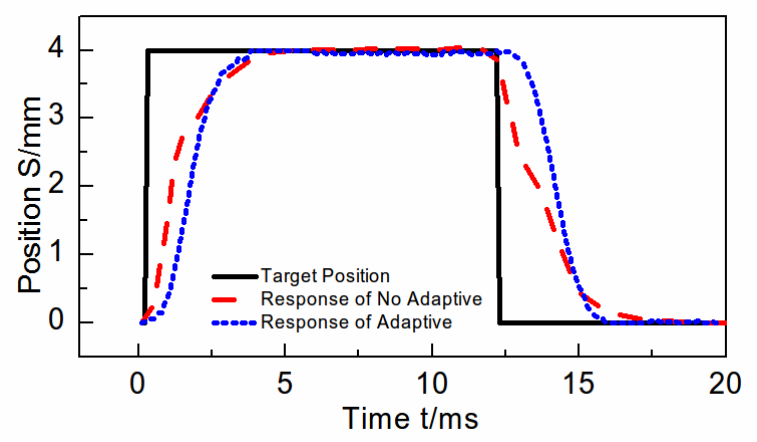

Figure 10. Verification of Adaptive Inverse System

For verification the adaptive control algorithm of inverse system, the actual value to the online real time identification is used to modify the actual inverse system control parameters. Its control effect is shown as Figure 10. It can be seen from Figure 10 that the 
location output has better robustness after the modification through parameter identification.

\section{Conclusion}

It identifies the parameter of EMLA by recursive least square, and bulids simulation model under Matlab/Simulink, and verifies the feasibility of identification algorithm, and can accurately identify EMLA's resistance, inductance, motion mass, viscous friction coefficient and the outside interference force. By using the experiment data collected it makes off-line identification, and combined with identification of real-time online adjustment. Meanwhile, the influence of parameters change on the system performance is simulated, thus the compensation method is proposed.

The identified parameter is passed to the inverse system control of EMLA, and achieves parameter adaptive control of online adjustment. The experiment concides with simulation results coincide. Through the control scheme of the adaptive parameter identification inverse system, it can improve the problem on the fall of the control system performance, which caused by the temperature rise with the increase of resistance while EMLA is in the long run, and improve the robustness for the system's long-runing.

\section{References}

[1] L. Liu and S. Q. Chang. "Improvement of valve seating performance of engine's electromagnetic valvetrain", Mechatronics, vol .21, no.7, (2011), pp. 1234-1238.

[2] D. S. Ribeiro, L. A. Jacobina, C. B. Lima and A. N. Oliveira, "Real-time estimation of the electric parameters of an induction machine using sinusoidal PWM voltage waveforms", IEEE Transactions on Industry Applications, vol.36, no.3, (2000), pp. 743-754.

[3] B. Karanayil, F. R. Muhammed and G. Colin, "Online stator and rotor resistance estimation scheme using artificial neural networks for vector controlled speed sensorless induction motor drive", IEEE Transactions on Industrial Electronics, vol. 54, no.1, (2007), pp. 167-176.

[4] W. H. Wang and X. Xiao, "Adaptive incremental predictive control method for current of PMSM based on online identification of inductance", Electric Machines \& Control/Dianji Yu Kongzhi Xuebao, vol. 18, no. 2, (2014), pp. 75-82.

[5] R. Krneta, S. Antić and D. Stojanović, "Recursive least squares method in parameters identification of DC motors models", Facta universitatis-series: Electronics and Energetics, vol. 18, no. 3 (2005), pp. 467-478.

[6] F. Muzi, "A filtering procedure based on least squares and Kalman algorithm for parameter estimate in distance protection", International Journal of Circuits, Systems and Signal Processing, vol. 1, no. 1 (2007), pp.16-21.

[7] R. Trabelsi, A. Khedher, M. F. Mimouni and F. M'sahli, "Backstepping control for an induction motor using an adaptive sliding rotor-flux observer”, Electric Power Systems Research, no. 93 (2012), pp. 115.

[8] D. P. Marcetic and S. N. Vukosavic, "Speed-sensorless AC drives with the rotor time constant parameter update", IEEE Transactions on Industrial Electronics, vol. 54, no.5, (2007), pp. 2618-2625.

[9] X. X. Shi, H. S. Li and J. C. Huang, "A practical scheme for induction motor modelling and speed control”, International Journal of Control \& Automation, vol. 7, no.4, (2014),pp. 113-124.

[10] V. V. Terzija and Z. M. Radojevic, "Numerical algorithm for adaptive autoreclosure and protection of medium-voltage overhead lines", IEEE Transactions on Power Delivery, vol. 19, no. 2, (2004), pp. 554559.

\section{Authors}

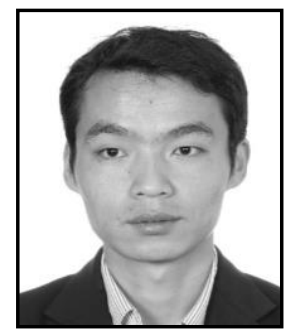

Jianhui Zhu, received the B.Eng. and Master degrees in mechatronic control engineering from Anhui Agriculture University, Hefei, China, in 2007 and 2010, and is pursuing the $\mathrm{Ph} . \mathrm{D}$. degree in mechatronic control engineering of Nanjing University of Science and Technology, Nanjing, China. His research interests include precision motion control of electromagnetic linear actuator, sensor and robotics. 


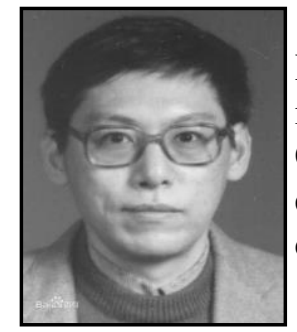

Siqin Chang, obtained a Ph.D. in Vehicle Engineering from Huazhong University of Science, Wuhan, China. Now he works in Nanjing University of Science and Technology, Nanjing, China. In the recent ten years, he has worked in the areas of electronic vehicle control system, mechatronics technology and control of electromagnetic linear actuator. 
International Journal of Control and Automation Vol.8, No.12 (2015) 